\title{
EFEITO DAS VARIAÇÕES BIOMÉTRICAS DE MUDAS CLONAIS DE EUCALIPTO SOBRE O CRESCIMENTO NO CAMPO ${ }^{1}$
}

Fábio Afonso Mazzei Moura de Assis Figueiredo², José Geraldo de Araújo Carneiro ${ }^{3}$, Ricardo Miguel Penchel $^{4}$, Deborah Guerra Barroso ${ }^{5}$ e Rogério Figueiredo Daher ${ }^{5}$

\begin{abstract}
RESUMO - Este trabalho objetivou avaliar o efeito da variação de altura e diâmetro de colo de mudas clonais do híbrido de Eucalyptus grandis x E. urophylla sobre o crescimento após o plantio nos sítios de Aracruz-ES, Mucuri-BA, Montanha-ES e Carlos Chagas-MG. As classes selecionadas foram: mudas altas e grossas (AG), mudas altas com diâmetro médio (AM), mudas médias com diâmetro grosso (MG), mudas médias com diâmetro médio (MM), mudas pequenas com diâmetro médio (PM) e mudas pequenas com diâmetro fino (PF). Os valores dessas classes foram: altura: A (31-40 cm), M (21-30 cm) e P (10-20 cm); diâmetro: G (3,1-4,0 mm), M (2,1-3,0 mm) e F (1,5-2,0 mm). As mudas, 90 dias após o transplantio nos tubetes e consideradas rustificadas, foram plantadas e as avaliações de altura (H) e diâmetro (D), feitas no $1^{\circ}, 2^{\circ}, 3^{\circ}, 6^{\circ}$ e $12^{\circ}$ meses. A massa de matéria seca de folhas, lenho, casca, galhos e raízes finas, médias e grossas foram avaliadas no sexto mês. Mudas com maiores dimensões de altura e diâmetro apresentaram maior crescimento inicial, mas as diferenças tenderam a diminuir ao longo do tempo. O sítio de Carlos Chagas teve as piores condições para o crescimento das plantas.
\end{abstract}

Palavras-chave: Qualidade de mudas, Características morfológicas, Mudas clonais e Eucalyptus spp.

\section{BIOMETRIC VARIATION EFFECT OF EUCALIPT CLONAL CUTTINGS ON THE GROWTH IN THE FIELD}

\begin{abstract}
This work aimed to evaluate the height and root collar diameter effects of the hybrid Eucalyptus grandis $x$ E. urophylla cuttings on the growth after planting in the Aracruz-ES, Mucuri-BA, Montanha-ES and Carlos Chagas-MG sites. The selected classes were: high cuttings with thick root collar diameter (AG), high cuttings with averaged size diameter (AM), average height with thick diameter (MG), average height and average diameter (MM), low cutting with average size diameter (PM) and low cutting with fine diameter roots (PF). The values of the classes were: height- $A(31-40 \mathrm{~cm}), M(21-30 \mathrm{~cm})$ and $P(10-20 \mathrm{~cm})$; root collar diameter$G(3.1-4.0 \mathrm{~cm}), M(2.1-3.0 \mathrm{~mm})$ and $F(1.5-2.0 \mathrm{~mm})$. The cuttings, 90 days after transplanting to tubes, were taken as hardened and outplanted. The evaluations were in height (A), diameters (D) were measured at the 1st, 2nd, 3rd, 6th and 12th months. Leaf dry mass, wood, bark, branches and fine, average size and thick roots were evaluated at the 6th month. Higher and thicker cutting presented higher initial growth, although the differences showed decreasing values over time. Carlos Chagas showed the worst growth conditions.
\end{abstract}

Keywords: Cutting quality, Morphological characteristics, Clonal cutting and Eucalyptus spp.

\footnotetext{
${ }^{1}$ Recebido em 15.05.2008 e aceito para publicação em 25.08.2010.

${ }^{2}$ Doutorado em andamento pela Universidade Estadual do Norte Fluminense Darcy Ribeiro, UENF, Brasil. E-mail:

<fabio_uenf@yahoo.com.br>.

${ }^{3}$ Universidade Estadual do Norte Fluminense Darcy Ribeiro, UENF, Brasil. E-mail:<carneiro@uenf.br>.

${ }^{4}$ Centro de tecnologia FIBRIA Celulose. E-mail: <rp@fibria.com.br>.

${ }^{5}$ Universidade Estadual do Norte Fluminense Darcy Ribeiro. E-mail: <deborah@uenf.br>e <rogdaher@uenf.br>.
} 


\section{INTRODUÇÃO}

O setor florestal começou a expandir-se, significativamente, a partir dos incentivos fiscais, na década de 1960, o que proporcionou reflorestamentos extensivos, para diminuir o desmatamento (RESENDE et al., 1996), constituindo iniciativa fundamental para a redução da demanda por espécies nativas.

O setor florestal contribui com 5\% do PIB nacional (US\$ 25,9 bilhões), sendo responsável, também, por gerar 1,6 milhão de empregos diretos e 5,6 milhões de empregos indiretos (SOCIEDADE BRASILEIRA DE SILVICULTURA, 2006).

As pesquisas têm buscado novas tecnologias, visando estabelecer florestas mais produtivas. Segundo Santos et al. (2000), o crescente aumento do consumo de produtos florestais indica a necessidade da utilização de espécies de rápido crescimento.

O gênero Eucalyptus é um dos mais adotados nos programas de reflorestamento no Brasil, por apresentar características de rápido crescimento e de boa adaptação às diferentes condições edafoclimáticas (BERGER et al., 2002).

A produção das mudas é de suma importância para o sucesso da atividade, pois, segundo Carneiro (1995), o seu percentual de sobrevivência e crescimento, após o plantio estão diretamente relacionados à sua qualidade.

As qualidades morfológica e fisiológica das mudas dependem da constituição genética, das condições ambientais, dos métodos de produção, do regime de manejo e dos cuidados no transporte e no plantio (PARVIAINEN, 1981).

Atualmente, a produção de mudas de eucalipto é feita, principalmente, por meio da clonagem, que procura assegurar a manutenção das características da plantamatriz-elite selecionada e a implantação de talhões uniformes de elevada produtividade (ALFENAS et al., 2004). Mesmo sendo a clonagem técnica que permite a manutenção das características desejadas em uma planta, ocorrem, em virtude do manejo, variações no crescimento de mudas no viveiro e no campo.

A expedição de mudas de qualidade contribuirá para a redução de custos com o replantio, e o crescimento mais acelerado poderá contribuir para que superem a competição com as plantas daninhas mais rapidamente, promovendo a diminuição de gastos com tratos culturais.
Este trabalho objetivou avaliar o efeito da variação de altura e diâmetro de mudas clonais do híbrido de Eucalyptus grandis com E. urophylla sobre o crescimento após o plantio em sítios de diferentes condições edafoclimáticas, localizados em Aracruz-ES, Mucuri-BA, Montanha-ES, e Carlos Chagas-MG.

\section{MATERIAL E MÉTODOS}

O trabalho envolveu duas etapas: a primeira, referente à produção e distribuição das mudas em classes de altura e diâmetro de colo, realizada 90 dias após a estaquia, desenvolvida no viveiro da empresa FIBRIA, no Município de Aracruz, ES. Na segunda etapa, as mudas foram expedidas para o plantio, em quatro sítios: Aracruz (região central do Espírito Santo, latitude de 1948' S e longitude de $40^{\circ} 17^{\prime}$ W W altitude de $60 \mathrm{~m}$ ), Mucuri (região Sul da Bahia, a $18^{\circ} 16^{\prime} \mathrm{S}$ e $39^{\circ} 41^{\prime} \mathrm{W}$ e altitude de $7 \mathrm{~m}$ ), Montanha (Norte do Espírito Santo, a $18^{\circ} 07^{\prime} \mathrm{S}$ e $40^{\circ} 21^{\prime} \mathrm{W}$ e altitude de $150 \mathrm{~m}$ ) e Carlos Chagas (Norte de Minas Gerais, a $17^{\circ} 42^{\prime}$ S e $40^{\circ} 45^{\prime}$ W e 160 m de altitude).

De acordo com a classificação climática proposta por Köppen, os sítios de plantio são classificados em: Aracruz e Mucuri como Am e os sítios de Montanha e Carlos Chagas como Aw.

Na Tabela 1 são apresentadas as médias mensais de temperatura, umidade relativa do ar e precipitação pluviométrica durante o período de avaliação do experimento.

O deficit de pressão de vapor (DPV), calculado segundo a equação proposta por Naves-Barbiero et al. (2000), por meio das suas médias mensais nos diferentes sítios de plantio, é apresentado na Figura 1.

Os solos dos sítios são classificados como: Aracruz, onde predominam o Latossolo Vermelho-Amarelo distrófico coeso + Podzólico Vermelho-Amarelo distrófico abrupto; Mucuri, onde predomina o Latossolo Amarelo distrófico, com manchas de solos arenosos no horizonte superficial; Montanha, onde predominam o Latossolo Vermelho-Amarelo distrófico + Podzólico VermelhoAmarelo distrófico; Carlos Chagas, onde se observa a predominância de Latossolo Vermelho-Amarelo distrófico. 
Tabela 1 - Médias mensais de temperatura $\left(\mathrm{T}^{\circ} \mathrm{C}\right.$ ) e umidade relativa do ar (UR\%) e precipitação (Prec.) nos diferentes sítios de plantio, durante o período de avaliação após o plantio.

Table 1 - Monthly temperature average $\left(T^{\circ} \mathrm{C}\right)$ and air relative moisture content (UR\%), rainfall (Prec.) in the different planting sites during the evaluating period after the planting.

\begin{tabular}{|c|c|c|c|c|c|c|c|c|c|c|c|c|}
\hline \multirow[b]{2}{*}{ Mês } & \multicolumn{3}{|c|}{ Aracruz } & \multicolumn{3}{|c|}{ Mucuri } & \multicolumn{3}{|c|}{ Montanha } & \multicolumn{3}{|c|}{ Carlos Chagas } \\
\hline & $\mathrm{T}^{\circ} \mathrm{C}$ & UR\% & $\begin{array}{l}\text { Prec. } \\
(\mathrm{mm})\end{array}$ & $\mathrm{T}^{\circ} \mathrm{C}$ & UR\% & $\begin{array}{l}\text { Prec. } \\
(\mathrm{mm})\end{array}$ & $\mathrm{T}^{\circ} \mathrm{C}$ & UR\% & $\begin{array}{l}\text { Prec. } \\
(\mathrm{mm})\end{array}$ & $\mathrm{T}^{\circ} \mathrm{C}$ & UR\% & $\begin{array}{l}\text { Prec. } \\
(\mathrm{mm})\end{array}$ \\
\hline 1 & 23.5 & 64.3 & 22.1 & 23.1 & 80.0 & 66.0 & 25.2 & 87.3 & 129.7 & 31.6 & 71.7 & 140.0 \\
\hline 2 & 22.8 & 70.2 & 127.2 & 24.1 & 81.2 & 131.5 & 26.8 & 83.1 & 296.1 & 33.8 & 73.2 & 190.0 \\
\hline 3 & 23.7 & 71.6 & 142.5 & 24.9 & 82.5 & 250.9 & 26.8 & 85.6 & 124.9 & 34.1 & 75.2 & 210.0 \\
\hline 4 & 24.4 & 74.6 & 237.2 & 25.6 & 82.5 & 154.9 & 25.3 & 82.0 & 192.5 & 31.6 & 78.9 & 215.0 \\
\hline 5 & 25.3 & 73.9 & 192.0 & 25.3 & 83.7 & 234.4 & 25.3 & 85.0 & 145.2 & 31.5 & 56.2 & 95.0 \\
\hline 6 & 25.1 & 80.6 & 249.4 & 25.6 & 85.9 & 237.2 & 24.4 & 89.9 & 25.9 & 29.8 & 47.1 & 80.0 \\
\hline 7 & 25.4 & 83.5 & 236.8 & 24.8 & 85.0 & 37.0 & 22.9 & 89.3 & 110.4 & 29.3 & 72.1 & 120.0 \\
\hline 8 & 24.8 & 81.2 & 66.0 & 23.4 & 86.2 & 169.9 & 21.4 & 92.5 & 115.3 & 27.1 & 54.6 & 60.0 \\
\hline 9 & 22.9 & 82.6 & 335.5 & 22.0 & 88.4 & 164.8 & 21.9 & 86.0 & 20.3 & 27.3 & 53.9 & 55.0 \\
\hline 10 & 21.5 & 84.7 & 160.0 & 21.2 & 84.2 & 41.4 & 22.6 & 86.5 & 71.1 & 28.1 & 59.3 & 60.0 \\
\hline 11 & 20.7 & 80.8 & 82.3 & 22.0 & 83.8 & 103.1 & 23.1 & 84.4 & 35.8 & 28.8 & 32.7 & 22.0 \\
\hline 12 & 21.9 & 81.4 & 26.4 & 22.5 & 84.0 & 66.5 & 24.8 & 77.0 & 18.8 & 32.1 & 41.5 & 28.0 \\
\hline
\end{tabular}

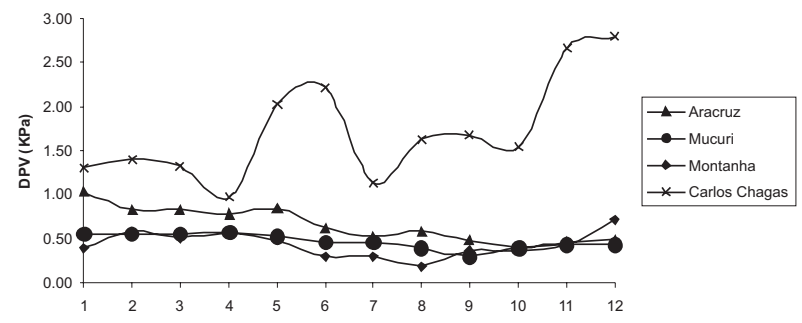

Figura 1 - Déficit de pressão de vapor do ar (DPV) durante 12 meses após a instalação do experimento nos sítios de plantio.

Figure 1 - Water vapor deficit of the air (DPV) during 12 months after setting up the experiment on the planting sites.

\subsection{Produção e classificação das mudas}

Mudas do clone 11 foram produzidas de acordo com o sistema convencional da empresa, com a utilização de miniestacas, variando entre 8 e $10 \mathrm{~cm}$, retiradas da posição intermediária na brotação das minicepas do jardim clonal.

O tipo de recipiente utilizado foi o tubete de polipropileno, com capacidade volumétrica de $53 \mathrm{~cm}^{3}$. O substrato foi composto pela mistura de $40(\mathrm{v} / \mathrm{v})$ de casca de eucalipto compostada +30 $(\mathrm{v} / \mathrm{v})$ de vermiculita expandida tipo fina $+30 \%(\mathrm{v} / \mathrm{v})$ de casca de arroz carbonizada. Ao substrato foram adicionados $2,0 \mathrm{~kg} \mathrm{~m}^{-3}$ de Osmocote ${ }^{\circledR}$ (19-06-10) microgranulado de liberação lenta $+2,0 \mathrm{kgm}^{-3}$ de superfosfato simples. O ciclo de produção das mudas durou aproximadamente 90 dias, desde o transplantio das miniestacas até a expedição das mudas para o campo.

A altura foi avaliada com régua milimetrada e o diâmetro, com paquímetro digital. As mudas foram divididas em três classes de altura: mudas altas, médias e pequenas. Dentro de cada classe de altura, as mudas foram divididas em três classes de diâmetro: diâmetros grossos, médios e finos. Assim, foram definidas nove classes de mudas: AG - mudas altas, com diâmetro grosso; AM - altas, com diâmetro médio; AF - altas, com diâmetro fino; MG - médias, com diâmetro grosso; MM - médias, com diâmetro médio; MF - médias, com diâmetro fino; PG - pequenas, com diâmetro grosso; PM - pequenas, com diâmetro médio; e PF - mudas pequenas, com diâmetro fino.

Foram consideradas mudas altas as que apresentaram valores acima do desvio de +1 sd da média, de altura média, aquelas com dimensões entre -1 sd e +1 sd da média e pequenas, as que apresentaram valores abaixo do desvio de -1 sd da média. O mesmo procedimento foi adotado para a divisão das mudas em classes de diâmetro. No entanto, durante a etapa de classificação não foi possível encontrar mudas que se enquadrassem nas classes AF, MF e PG.

Na Tabela 2 são apresentadas as dimensões das classes de mudas do clone estudado.

Revista Árvore, Viçosa-MG, v.35, n.1, p.01-11, 2011 
Tabela 2 - Intervalo das médias de altura e diâmetro de mudas do híbrido de Eucalyptus grandis x E. urophylla. Table 2 - Interval between height and root collar diameter averages of hybrid Eucalyptus grandis $x$ E. urophylla.

\begin{tabular}{ccc}
\hline Tratamento & Altura $(\mathrm{cm})$ & Diâmetro (mm) \\
\hline AG & 31 a 40 & 3,1 a 4,0 \\
AM & 31 a 40 & 2,1 a 3,0 \\
MG & 21 a 30 & 3,1 a 4,0 \\
MM & 21 a 30 & 2,1 a 3,0 \\
PM & 10 a 20 & 2,1 a 3,0 \\
PF & 10 a 20 & 1,5 a 2,0 \\
\hline
\end{tabular}

AG: mudas altas, com diâmetro grosso; AM: mudas altas, com diâmetro médio; MG: mudas médias, com diâmetro grosso; MM: mudas médias, com diâmetro médio; PM: mudas pequenas, com diâmetro médio; e PF: mudas pequenas, com diâmetro fino.

\subsection{Implantação do experimento e avaliações do crescimento}

Realizou-se o controle das formigas antes e depois do plantio, utilizando isca formicida granulada, sendo aplicados $10 \mathrm{~g} \mathrm{~m}^{-2}$ de terra solta do formigueiro.

Cinco dias antes do plantio, efetuou-se a primeira adubação, aplicando 300 g por cova, da formulação granulada de NPK (06-30-06) + 1,0\% de Zn + 0,3\% de Cu. Após 90 dias do plantio, fez-se adubação de cobertura, aplicando 150 g por cova, da formulação NPK (12-00-20) $+0,7 \%$ de $B$, na projeção da copa.

As mudas foram plantadas manualmente, entre meses de outubro e novembro de 2004, no espaçamento $3 \times 2$. Logo após o plantio, as mudas foram irrigadas, recebendo aproximadamente $8 \mathrm{~L}$ de água por cova.

As plantas foram monitoradas com relação à altura e diâmetro do colo aos um, dois e Três meses após o plantio. Aos seis e doze meses, além da altura, a medição de diâmetro foi realizada à altura do peito. Para a medição da altura das plantas mais baixas, utilizaram-se régua telescópica graduada fabricada pela Aracruz Celulose S. A. e clinômetro mas plantas mais altas. Mediu-se o diâmetro com fita diamétrica.

Seis meses após o plantio, procedeu-se às quantificações do peso de massa seca de folhas, galhos, casca, lenho e raízes grossas, médias e finas, separadamente, de uma planta representativa da altura e dos diâmetros médios, em cada parcela. Amostras dos componentes das plantas selecionadas foram levadas à estufa de circulação forçada de ar até atingir peso constante, a $105^{\circ} \mathrm{C}$, sendo, posteriormente, pesadas (BÖHM, 1979).

Para o estudo do crescimento das raízes, aos seis mese, fizeram-se escavações à distância de $1 \mathrm{~m}$, em torno da planta, até a profundidade alcançada pelas raízes, que foram cuidadosamente retiradas para as determinações do peso de massa seca. Para a determinação da biomassa, as raízes foram separadas em função do diâmetro, sendo consideradas grossas as raízes com diâmetro acima de $5 \mathrm{~mm}$, médias as com diâmetro entre 2,5 e 4,99 mm e finas aquelas raízes com diâmetro inferior a $2,49 \mathrm{~mm}$.

Na sequência, efetuou-se a análise de variância conjunta dos experimentos, sendo testados seis tratamentos, em quatro sítios, com três repetições e 25 plantas por parcela. O experimento foi implantado em sistema de blocos ao acaso. As médias foram comparadas pelo teste de Duncan a 5\% de probabilidade.

\section{RESULTADOS E DISCUSSÃO}

Na Tabela 3 são apresentadas as médias de altura da parte aérea e do diâmetro de mudas clonais no $1^{\circ}$, $2^{\circ}, 3^{\circ}, 6^{\circ}$ e $12^{\circ}$ mês após o plantio. Por questões operacionais, as medições no $1^{\circ}$ e no $12^{\circ}$ mês não puderam ser realizadas, no sítio de Aracruz.

A altura, até o terceiro mês, mostrou que os tratamentos apresentaram diferenças significativas, em função da classificação realizada no viveiro, à exceção do sítio de Carlos Chagas. Com referência ao diâmetro, nos dois primeiros meses apenas as plantas de Carlos Chagas mostraram equivalência entre os tratamentos, evidenciado o efeito da classificação nos demais sítios. No terceiro mês, os sítios apresentaram equivalência, excetuando-se o de Montanha. Esses resultados corroboram os obtidos por Barnett (1983), que constatou maior crescimento em altura no campo, à medida que as dimensões de altura das mudas, por ocasião do plantio, foram maiores (Tabela 3). Freitas (2003), avaliando o crescimento pós-plantio de clones de eucalipto, constatou que mudas clonais com maiores valores de altura e diâmetro apresentaram maior crescimento. Morgado

Revista Árvore, Viçosa-MG, v.35, n.1, p.01-11, 2011

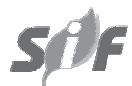


Tabela 3 - Altura e diâmetro de mudas clonais de Eucalyptus grandis x E. urophylla, em diferentes períodos após o plantio. Table 3 - Height and root collar diameter of clonal Eucalyptus gandis $x \boldsymbol{E}$ erophylla cuttings in different periods after the planting.

\begin{tabular}{|c|c|c|c|c|c|c|c|c|c|}
\hline $\begin{array}{l}\text { Tempo } \\
\text { (meses) }\end{array}$ & Trat & Aracruz & Mucuri & Montanha & $\begin{array}{l}\text { Carlos } \\
\text { Chagas }\end{array}$ & Aracruz & Mucuri & Montanha & $\begin{array}{l}\text { Carlos } \\
\text { Chagas }\end{array}$ \\
\hline & & \multicolumn{5}{|c|}{ Altura (m) } & \multicolumn{3}{|c|}{ Diâmetro do colo $(\mathrm{cm})$} \\
\hline \multirow[t]{7}{*}{1} & AG & - & 0.70 а $\mathrm{A}$ & $0.42 \mathrm{~b} \mathrm{~A}$ & 0.35 с A & - & 1.16 a A & $0.45 \mathrm{~b} \mathrm{~A}$ & 0.34 с A \\
\hline & $\mathrm{AM}$ & - & 0.68 а $\mathrm{AB}$ & 0.42 b A & 0.32 с АВ & - & 1.11 a $\mathrm{ABC}$ & $0.40 \mathrm{~b} \mathrm{AB}$ & 0.27 с A \\
\hline & MG & - & 0.64 а BC & 0.37 b B & 0.28 с ВC & - & 1.12 а $\mathrm{AB}$ & $0.41 \mathrm{~b} \mathrm{AB}$ & 0.29 с A \\
\hline & MM & - & 0.62 a CD & 0.32 b BC & 0.28 b BC & - & 1.03 a CD & 0.35 b B & $0.30 \mathrm{~b} \mathrm{~A}$ \\
\hline & $\mathrm{PM}$ & - & 0.59 a $\mathrm{DE}$ & 0.31 b C & 0.21 с D & - & 1.06 а BC & $0.37 \mathrm{~b} \mathrm{AB}$ & 0.27 с A \\
\hline & $\mathrm{PF}$ & - & 0.57 а $\mathrm{E}$ & $0.33 \mathrm{~b} \mathrm{BC}$ & 0.25 с CD & - & 0.97 a $\mathrm{D}$ & $0.37 \mathrm{~b} \mathrm{AB}$ & 0.25 с A \\
\hline & & \multicolumn{5}{|c|}{$\mathrm{CV}(\%)=6,45$} & \multicolumn{3}{|c|}{$\mathrm{CV}(\%)=8,10$} \\
\hline \multirow[t]{7}{*}{2} & AG & $0.80 \mathrm{~b} \mathrm{AB}$ & 0.91 a $\mathrm{A}$ & $0.75 \mathrm{~b} \mathrm{~A}$ & 0.50 с A & $1.43 \mathrm{~b} \mathrm{~A}$ & 1.88 а $\mathrm{A}$ & 1.10 с A & $0.55 \mathrm{~d} \mathrm{~A}$ \\
\hline & $\mathrm{AM}$ & 0.82 а $\mathrm{A}$ & 0.86 а $\mathrm{AB}$ & 0.74 b A & 0.44 с АB & $1.50 \mathrm{~b} \mathrm{~A}$ & 1.78 а $\mathrm{AB}$ & 1.02 с AB & $0.44 \mathrm{~d} \mathrm{~A}$ \\
\hline & MG & 0.80 a $\mathrm{AB}$ & 0.85 а $\mathrm{ABC}$ & 0.69 b AB & 0.41 с В & 1.48 b A & 1.79 a $\mathrm{AB}$ & 1.05 с АB & $0.49 \mathrm{~d} \mathrm{~A}$ \\
\hline & MM & 0.75 a BC & 0.81 а BC & 0.61 b C & 0.41 с В & $1.33 \mathrm{~b} \mathrm{AB}$ & 1.75 a $\mathrm{AB}$ & 0.80 с C & $0.50 \mathrm{~d} \mathrm{~A}$ \\
\hline & $\mathrm{PM}$ & 0.71 b C & 0.82 а BC & 0.63 с ВС & $0.34 \mathrm{~d} \mathrm{C}$ & $1.33 \mathrm{~b} \mathrm{AB}$ & 1.78 a $\mathrm{AB}$ & 0.87 с ВC & $0.43 \mathrm{~d} \mathrm{~A}$ \\
\hline & $\mathrm{PF}$ & $0.68 \mathrm{~b} \mathrm{C}$ & 0.78 a C & $0.63 \mathrm{~b} \mathrm{BC}$ & 0.33 с C & $1.23 \mathrm{~b} \mathrm{~B}$ & 1.63 а B & 0.97 с АBC & $0.41 \mathrm{~d} \mathrm{~A}$ \\
\hline & & \multicolumn{5}{|c|}{$\mathrm{CV}(\%)=5,74$} & \multicolumn{3}{|c|}{$\mathrm{CV}(\%)=12,84$} \\
\hline \multirow[t]{7}{*}{3} & AG & 1,55 a $\mathrm{AB}$ & 1,40 ab A & $1,33 \mathrm{~b} \mathrm{~A}$ & 0,77 с A & 2,93 a $\mathrm{A}$ & 2,47 b A & 2,48 b A & 1,15 с $\mathrm{A}$ \\
\hline & $\mathrm{AM}$ & 1,60 a $\mathrm{A}$ & $1,29 \mathrm{~b} \mathrm{AB}$ & $1,31 \mathrm{~b} \mathrm{AB}$ & 0,62 с A & 2,96 а $A$ & $2,30 \mathrm{~b} \mathrm{~A}$ & $1 \mathrm{~b}$ A & 0,89 с $\mathrm{A}$ \\
\hline & MG & 1,62 а $A$ & 1,31 b AB & 1,27 b AB & 0,60 с $\mathrm{A}$ & 3,01 a $A$ & $2 \mathrm{~b} \mathrm{~A}$ & $\mathrm{~b} A B$ & 0,95 с $\mathrm{A}$ \\
\hline & MM & 1,50 а $\mathrm{AB}$ & $1,21 \mathrm{~b} \mathrm{AB}$ & 1,06 b C & 0,72 с A & 2,90 a $A$ & $1 \mathrm{~b} \mathrm{~A}$ & $\mathrm{~b}$ BC & 1,15 с $\mathrm{A}$ \\
\hline & $\mathrm{PM}$ & 1,49 а $\mathrm{AB}$ & 1,28 b AB & 1,04 с C & $0,65 \mathrm{~d} A$ & 2,90 a $A$ & $8 \mathrm{~b} \mathrm{~A}$ & 3 с C & $1,06 \mathrm{~d} \mathrm{~A}$ \\
\hline & $\mathrm{PF}$ & 1,39 а $\mathrm{B}$ & 1,16 b B & 1,13 b BC & 0,68 с $\mathrm{A}$ & 2,73 a $\mathrm{A}$ & $2,09 \mathrm{~b} \mathrm{~A}$ & 2,07 b ABC & 1,10 с $\mathrm{A}$ \\
\hline & \multicolumn{6}{|c|}{$\mathrm{CV}(\%)=8,81$} & \multicolumn{3}{|c|}{$\mathrm{CV}(\%)=14,30$} \\
\hline \multirow[t]{7}{*}{6} & AG & 4,17 а $\mathrm{AB}$ & 3,50 b A & 3,26 b A & 2,37 с $\mathrm{AB}$ & 3,56 а $A$ & 3,30 a $\mathrm{A}$ & 3,21 a $\mathrm{A}$ & $2,37 \mathrm{~b} \mathrm{AB}$ \\
\hline & $\mathrm{AM}$ & 4,43 a $\mathrm{AB}$ & 3,32 b AB & 3,23 b A & 2,11 с В & 3,62 a $A$ & $2,78 \mathrm{~b} \mathrm{AB}$ & $3,23 \mathrm{ab} A$ & 1,96 с B \\
\hline & MG & 4,50 a $A$ & 3,22 b AB & $3,18 \mathrm{~b} \mathrm{AB}$ & 2,32 с $\mathrm{AB}$ & 3,72 a $A$ & $2,91 \mathrm{~b} \mathrm{AB}$ & $3,14 \mathrm{~b} \mathrm{~A}$ & 2,19 с $\mathrm{AB}$ \\
\hline & MM & 4,21 a $\mathrm{AB}$ & $2,94 \mathrm{~b} \mathrm{~B}$ & $2,77 \mathrm{~b} \mathrm{BC}$ & $2,60 \mathrm{~b} \mathrm{~A}$ & 3,55 a $A$ & $2,50 \mathrm{~b} \mathrm{~B}$ & $2,52 \mathrm{~b} \mathrm{~B}$ & $2,57 \mathrm{~b} \mathrm{~A}$ \\
\hline & $\mathrm{PM}$ & 4,41 a $\mathrm{AB}$ & $3,28 \mathrm{~b} \mathrm{AB}$ & 2,66 с C & 2,49 с $\mathrm{AB}$ & 3,63 a $A$ & $2,93 \mathrm{~b} \mathrm{AB}$ & 2,35 с В & $2,26 \mathrm{c} \mathrm{AB}$ \\
\hline & $\mathrm{PF}$ & 4,01 a $\mathrm{B}$ & $2,91 \mathrm{~b} \mathrm{~B}$ & $2,91 \mathrm{~b}$ ABC & 2,47 с $\mathrm{AB}$ & 3,28 a $A$ & 2,39 b B & $2,72 \mathrm{~b} \mathrm{AB}$ & $2,30 \mathrm{~b} \mathrm{AB}$ \\
\hline & & \multicolumn{5}{|c|}{$\mathrm{CV}(\%)=7,17$} & \multicolumn{3}{|c|}{$\mathrm{CV}(\%)=10,41$} \\
\hline \multirow[t]{7}{*}{12} & AG & - & 8,17 a $\mathrm{B}$ & 8,50 a $\mathrm{A}$ & $5,74 \mathrm{~b} \quad \mathrm{~A}$ & - & 7,50 a $\mathrm{A}$ & 7,63 а $\mathrm{AB}$ & $6,13 \mathrm{~b} \mathrm{AB}$ \\
\hline & $\mathrm{AM}$ & - & 8,66 а $\mathrm{AB}$ & 8,53 a $A$ & $5,60 \mathrm{~b} \quad \mathrm{~A}$ & - & 6,95 b $\mathrm{B}$ & 7,83 а $\mathrm{A}$ & 5,72 с $\quad$ B \\
\hline & MG & - & 9,33 а $\mathrm{A}$ & 8,35 b A & 5,97 с A & - & 7,04 b B & 7,68 а $\mathrm{AB}$ & 6,21 с A \\
\hline & MM & - & 8,29 а $\mathrm{B}$ & 7,97 a $\mathrm{A}$ & $6,23 \mathrm{~b} \quad \mathrm{~A}$ & - & 6,47 b CD & 7,16 a $\mathrm{C}$ & $6,52 \mathrm{~b} \quad \mathrm{~A}$ \\
\hline & $\mathrm{PM}$ & - & 8,59 а $\mathrm{B}$ & 8,05 a $A$ & $6,07 \mathrm{~b} \quad \mathrm{~A}$ & - & 6,92 а $\mathrm{BC}$ & 7,24 а $\mathrm{BC}$ & $6,27 \mathrm{~b} \quad \mathrm{~A}$ \\
\hline & $\mathrm{PF}$ & - & 8,55 а $\mathrm{AB}$ & 8,20 a $A$ & $6,07 \mathrm{~b} \quad \mathrm{~A}$ & - & 6,29 b D & 7,39 а $\mathrm{ABC}$ & $6,30 \mathrm{~b} \quad \mathrm{~A}$ \\
\hline & \multicolumn{6}{|c|}{$\mathrm{CV}(\%)=6,29$} & \multicolumn{3}{|c|}{$\mathrm{CV}(\%)=3,66$} \\
\hline
\end{tabular}

Médias seguidas pelas mesmas letras minúsculas na linha e maiúscula na coluna não diferem entre si, pelo teste de Duncan a $5 \%$ de probabilidade.

Para o clone 11097: mudas altas (A) 31 a 40 cm; médias (M) 21 a 30 cm; pequenas (P) 10 a 20 cm; mudas grossas (G) 3,1 a 4,0 mm; médias (M) 2,1 a $3 \mathrm{~mm}$ e finas (F) 1,5 a 2,0 mm.

(2000), Leles (2000) e Barroso et al. (2000a-c), mostraram, também, que mudas de Eucalyptus spp. produzidas por sementes que tiveram maior altura e diâmetro no viveiro foram responsáveis por maior crescimento após o plantio.
Quanto mais rápido as plantas de eucalipto superarem a competição com as plantas invasoras, maior será a eficiência no uso de luz, água e nutrientes. De acordo com Carneiro (1995), o padrão de qualidade de mudas varia entre espécies e, em uma mesma espécie, entre sítios.

Revista Árvore, Viçosa-MG, v.35, n.1, p.01-11, 2011 
Seis meses após o plantio, para o crescimento em altura, ainda persistem algumas diferenças, entre tratamentos, em todos os sítios. Deve-se ressaltar a diferença, em Mucuri, entre as médias de AG e PF. Esses resultados convergem com o apresentado por Borges et al. (1980), que relataram correlações positivas entre altura das mudas de Eucalyptus grandis no viveiro e no campo, seis meses após o plantio, sendo compatíveis com os encontrados neste trabalho, na maioria dos sítios e tratamentos. Com referência ao crescimento diametral, as observações corresponderam ao constatado para a altura, excetuando-se o sítio de Aracruz (Tabela 3).

A altura nos sítios de Montanha e Carlos Chagas ao $12^{\circ}$ mês não evidenciaram diferenças significativas. Em Mucuri, constataram-se diferenças significativas, e, embora tenha apresentado a maior média, o tratamento MG não diferiu dos AM e PF.

Os resultados apresentados no $12^{\circ}$ mês estão de acordo com o que foi mostrado por Carneiro e Ramos (1981), trabalhando com Pinus taeda. Esses autores constataram equivalência em crescimento seis anos após o plantio, partindo-se de mudas com diferentes padrões de qualidade. Da mesma forma, José et al. (2005), trabalhando com Schinus terebinthifolius (aroeira), verificaram que as diferenças iniciais de altura e diâmetro das mudas tendem a desaparecer, ao longo do tempo, no campo.

Comparações entre os diferentes sítios de plantio, nos diferentes períodos de avaliação, evidenciaram diferenças de comportamento de crescimento em altura e diâmetro, o que demonstra a influência deles sobre o crescimento das plantas (Tabela 3).

A disponibilidade de água, temperatura e umidade relativa do ar afeta o crescimento das plantas pelo fato de sses elementos atuarem no controle da abertura e fechamento estomáticos e, consequentemente, a produção de biomassa seca. A redução na disponibilidade de água no solo resulta em diminuição do potencial hídrico foliar, visto que suas células não se encontram mais túrgidas, contribuindo para diminuição da condutância estomática e promovendo o fechamento parcial ou total dos estômatos (LARCHER, 2000; TAIZ; ZEIGER, 2004). Com o fechamento estomático, ocorre bloqueio no influxo de CO2 para as folhas, o que pode acarretar na paralisação do crescimento e perda de produtividade (Tabela 1; Figura 1).

Revista Árvore, Viçosa-MG, v.35, n.1, p.01-11, 2011

Revista Árvore, Viçosa-MG, v.35, n.1, p.01-11, 2011
Na Tabela 4 são apresentadas as médias do peso de massa de matéria seca de folhas (PSF), do lenho (PSL), da casca (PSC) e de galhos (PSG) de plantas clonais de eucalipto, seis meses após o plantio.

Com relação ao PSF, PSC e PSG, observou-se equivalência entre os tratamentos nos sítios de Mucuri, Montanha e Carlos Chagas. O sítio de Aracruz apresentou diferenças significativas entre as médias de alguns tratamentos, observando-se menores médias em PF. O PSL apresentou equivalência nos sítios de Aracruz, Mucuri e Carlos Chagas. Contudo, o sítio de Montanha apresentou diferenças significativas, sendo a maior média observada no tratamento AG. O sítio de Carlos Chagas teve as piores condições para produção de biomassa em todos os parâmetros avaliados. O PSL não mostrou diferenças significativas entre os tratamentos, exceto para AG e AM em Montanha. O tratamento AG foi responsável por maior produção do PSL de 42,83\% em Aracruz, de 59,83\% em Mucuri, de 102,48\% em Montanha e de 65,78\% em Carlos Chagas, em relação ao tratamento PF. De forma semelhante, Freitas (2003), trabalhando com o plantio de mudas de eucalipto com diferentes valores de altura e diâmetro de colo, constatou seis meses após o plantio, que, embora estatisticamente equivalentes, os valores absolutos foram consideráveis, sendo as mudas de maiores dimensões responsáveis por uma produção de $30 \%$ maior de lenho.

Considerando a importância da produção de lenho para as empresas, esta é uma das principais características avaliadas para a seleção de materia. Os ganhos também observados na produção de folhas, casca e galhos são importantes por dois motivos: a) quando deixados no local de colheita, para a ciclagem de nutrientes; b) se levado para o pátio da empresa, a casca, decomposta, poderá ser usada na produção de substrato ou geração de energia. Em relação à produção de massa seca de casca, o tratamento AG foi responsável pela produção de 41,21\% em Aracruz, 40,77\% em Mucuri, 4,4\% em Montanha e 64,83\% em Carlos Chagas, de biomassa a mais do que os valores apresentados pelo tratamento PF. Diferenças matemáticas de valores elevados também foram observadas para peso de matéria seca de folhas e galhos. No caso das folhas, maior peso de matéria seca pode estar relacionado à maior área foliar e, consequentemente, a maiores taxas fotossintéticas globais, contribuindo para maior crescimento das plantas (Tabela 4). 
Tabela 4 - Massa seca da parte aérea de plantas clonais de Eucalyptus grandis x E. urophylla, em diferentes sítios, seis meses após o plantio.

Table 4 - Stem dry matter of the clonal Eucalyptus grandis $x$ E. urophylla plants .in different sites six months after the planting.

\begin{tabular}{|c|c|c|c|c|}
\hline \multicolumn{5}{|c|}{ PSF (g) } \\
\hline Tratamento & Aracruz & Mucuri & Montanha & Carlos Chagas \\
\hline AG & 1623.20 a $\mathrm{AB}$ & 1304.81 a $\mathrm{A}$ & 1499.49 а A & 1153.78 а A \\
\hline $\mathrm{AM}$ & 1923.43 а A & 1145.38 bс A & $1602.94 \mathrm{ab} A$ & 622.15 с A \\
\hline MG & 1983.67 а A & 1140.56 b A & 1276.14 b A & 863.84 b A \\
\hline MM & 1472.64 а АB & 994.45 a A & 1338.87 а $\mathrm{A}$ & 1051.84 a A \\
\hline $\mathrm{PM}$ & 1662.01 а $\mathrm{AB}$ & 997.90 ab A & $1306.29 \mathrm{ab} \mathrm{A}$ & 648.44 b A \\
\hline $\mathrm{PF}$ & 1106.92 а B & 964.54 a $\mathrm{A}$ & 1377.91 а $\mathrm{A}$ & 815.12 a $\mathrm{A}$ \\
\hline \multicolumn{5}{|c|}{$\mathrm{CV}(\%)=31,95$} \\
\hline \multicolumn{5}{|c|}{ PSL (g) } \\
\hline Tratamento & Aracruz & Mucuri & Montanha & Carlos Chagas \\
\hline AG & $1013.05 \mathrm{ab} \mathrm{A}$ & 939.35 ab A & 1606.33 a A & 578.31 b A \\
\hline $\mathrm{AM}$ & 1191.83 а A & $676.21 \mathrm{ab} A$ & 1016.63 а $\mathrm{AB}$ & $112.68 \mathrm{~b} \mathrm{~A}$ \\
\hline MG & 1351.24 a $\mathrm{A}$ & 751.31 ab A & 821.78 ab B & 393.82 b A \\
\hline MM & 801.13 a $\mathrm{A}$ & 609.02 a $\mathrm{A}$ & 740.54 а B & 530.32 а $\mathrm{A}$ \\
\hline $\mathrm{PM}$ & 1183.78 а $\mathrm{A}$ & 768.37 ab A & 702.49 ab B & 356.42 b A \\
\hline $\mathrm{PF}$ & 709.26 а A & 587.69 а A & 793.29 а B & 348.83 а A \\
\hline \multicolumn{5}{|c|}{$\mathrm{CV}(\%)=52,01$} \\
\hline \multicolumn{5}{|c|}{ PSC (g) } \\
\hline Tratamento & Aracruz & Mucuri & Montanha & $\begin{array}{l}\text { Carlos Chagas } \\
\end{array}$ \\
\hline AG & 201.44 a $\mathrm{AB}$ & 196.58 a A & 181.56 a A & 133.47 a A \\
\hline AM & 245.17 a A & 156.37 a A & 207.88 а $A$ & $62.92 \mathrm{~b} \mathrm{~A}$ \\
\hline MG & 248.69 a A & 167.47 ab A & $173.02 \mathrm{ab} \mathrm{A}$ & 86.96 b A \\
\hline MM & 173.44 a $\mathrm{AB}$ & 144.59 а A & 154.39 а A & 114.38 a $\mathrm{A}$ \\
\hline $\mathrm{PM}$ & 225.98 а $\mathrm{AB}$ & $167.94 \mathrm{ab} \mathrm{A}$ & $153.01 \mathrm{ab} \mathrm{A}$ & $82.02 \mathrm{~b} \mathrm{~A}$ \\
\hline $\mathrm{PF}$ & 142.65 а B & 139.64 а A & 173.90 а $\mathrm{A}$ & 80.97 a A \\
\hline \multicolumn{5}{|c|}{ CV $(\%)=33,60$} \\
\hline \multicolumn{5}{|c|}{ PSG (g) } \\
\hline Tratamento & Aracruz & Mucuri & Montanha & Carlos Chagas \\
\hline AG & 1195.98 а $\mathrm{AB}$ & 1035.77 a $\mathrm{A}$ & 1240.70 a $\mathrm{A}$ & 913.45 a A \\
\hline $\mathrm{AM}$ & 1526.59 a $\mathrm{A}$ & 926.69 ab A & 1422.66 a $\mathrm{A}$ & 440.76 b A \\
\hline MG & 1642.36 а A & $952.09 \mathrm{~b} \mathrm{~A}$ & $1042.14 \mathrm{~b} \mathrm{~A}$ & $715.20 \mathrm{~b} \mathrm{~A}$ \\
\hline MM & 1196.18 а АВ & 817.02 a $\mathrm{A}$ & 1135.55 a A & 821.34 a A \\
\hline $\mathrm{PM}$ & 1403.00 а $A B$ & 859.76 ab A & 1162.68 a $\mathrm{A}$ & 483.57 b A \\
\hline $\mathrm{PF}$ & 842.40 а B & 774.54 a A & 1156.14 a $\mathrm{A}$ & 579.97 a A \\
\hline
\end{tabular}

Médias seguidas pelas mesmas letras minúsculas na linha e maiúscula na coluna não diferem entre si, pelo teste de Duncan a $5 \%$ de probabilidade.

Mudas altas (A) 31 a 40 cm; médias (M) 21 a 30 cm; pequenas (P) 10 a 20 cm; mudas grossas (G) 3,1 a 4,0 mm; médias (M) 2,1 a $3 \mathrm{~mm}$ e finas (F) 1,5 a $2,0 \mathrm{~mm}$.

Na Tabela 5 são apresentadas as médias de profundidade alcançada pelo sistema radicular, verificada seis meses após o plantio.

Em relação à profundidade alcançada pelo sistema radicular das plantas, constatou-se equivalência entre os tratamentos nos sítios de Aracruz, Mucuri e
Montanha. Contudo, o sítio de Carlos Chagas apresentou diferenças significativas entre os diferentes tratamentos, sendo a maior média alcançada pelo tratamento AG. Comparações entre os diferentes sítios não evidenciaram diferenças significativas para a maioria dos tratamentos (Tabela 5). Segundo Larcher (2000), 
Tabela 5 - Profundidade do sistema radicular de plantas clonais de Eucalyptus grandis x Eucalyptus urophylla, em diferentes sítios, seis meses após o plantio.

Table 5 - Root system depth of the hybrid Eucalyptus grandis $x$ E. urophylla plants on different sites, six months after planting.

\begin{tabular}{|c|c|c|c|c|}
\hline \multicolumn{5}{|c|}{ Profundidade $(\mathrm{cm})$} \\
\hline Tratamento & Aracruz & Mucuri & Montanha & Carlos Chagas \\
\hline AG & 112.66 b A & $92.66 \mathrm{~b} \mathrm{~A}$ & 105.66 b A & 170.66 a $\mathrm{A}$ \\
\hline $\mathrm{AM}$ & 110.00 a $\mathrm{A}$ & 73.33 а A & 89.00 a A & 112.33 а B \\
\hline MG & 99.00 a $\mathrm{A}$ & 106.33 a $\mathrm{A}$ & 68.66 a $\mathrm{A}$ & 116.00 a B \\
\hline MM & 110.00 a $\mathrm{A}$ & 72.33 а A & 99.66 а A & 120.00 а $\mathrm{AB}$ \\
\hline PM & 81.00 a A & 87.00 a $\mathrm{A}$ & 95.00 a A & 100.00 а B \\
\hline $\mathrm{PF}$ & $81.33 \mathrm{ab} A$ & 64.66 b A & 113.66 a $\mathrm{A}$ & 126.00 а $\mathrm{AB}$ \\
\hline \multicolumn{5}{|c|}{$C V(\%)=32,63$} \\
\hline
\end{tabular}

Médias seguidas pelas mesmas letras minúsculas na linha e maiúscula na coluna não diferem entre si, pelo teste de Duncan a de $5 \%$ de probabilidade.

Mudas altas (A) 31 a $40 \mathrm{~cm}$; médias (M) 21 a $30 \mathrm{~cm}$; pequenas (P) 10 a $20 \mathrm{~cm}$; mudas grossas (G) 3,1 a 4,0 mm; médias (M) 2,1 a $3 \mathrm{~mm}$; e finas (F) 1,5 a 2,0 $\mathrm{mm}$.

a distribuição e densidade de raiz dependem do tipo do sistema radicular e variam no decorrer do ano. O padrão e extensão do crescimento do sistema radicular são reflexos do controle genético e das características ambientais, com acentuadas influências das condições edáficas.

De acordo com Vogt e Persson (1991), o crescimento de raízes pode ser responsável por até 50\% da produtividade primária local, constituindo, dessa forma, um dos caminhos principais pelo qual o carbono entra no solo, sendo essencial para a continuidade e estabilidade da microbiota e para a ciclagem de nutrientes no ecossistema florestal.

Na Tabela 6 são apresentadas as médias do peso de massa de matéria seca de raízes finas (PSRF), médias (PSRM) e grossas (PSRG) do clone em estudo, seis meses após o plantio.

Em relação à massa seca de raízes finas, médias e grossas, apenas no sítio de Aracruz foram verificadas diferenças significativas entre os diferentes tratamentos. Em relação ao PSRF, a maior média foi apresentada pelo tratamento MG, mas não diferindo dos tratamentos MM e PM. A comparação entre os diferentes sítios evidenciou que no sítio de Aracruz os tratamentos apresentaram as maiores médias em relação aos demais. Quanto ao PSRM, também no sítio de Aracruz a maior média foi apresentada pelo tratamento AG contudo equivalente a MG, embora a diferença numérica tenha sido de 118,46\%. Ainda em relação ao PSRM, não foram observadas diferenças significativas entre os sítios de plantio, com exceção do tratamento AG, tendo o sítio de Aracruz apresentado produção superior de 197,13; 549,61; e 449,96\%, em relação aos sítios de Mucuri, Montanha e Carlos Chagas, respectivamente. Com referência ao PSRG, no sítio de Aracruz a maior média foi apresentada pelo tratamento MG, mas não diferiu apenas do tratamento PF. Foram verificadas diferenças significativas entre os sítios de plantio, sendo, de forma geral, as maiores médias exibidas no sítio de Aracruz e as menores no sítio de Carlos Chagas (Tabela 6).

Solo e variações de temperatura, umidade e precipitação contribuem para explicar a diferença de comportamento dos tratamentos nos diferentes sítios de plantio. De acordo com Souza et al. (2006), a radiação solar, temperatura e disponibilidade hídrica são os principais fatores climáticos que afetam o crescimento das espécies florestais. Dessa forma, o déficit hídrico constitui uma das maiores limitações da produtividade vegetal. No sítio de Carlos Chagas, a precipitação pluviométrica, a partir do quinto mês, ficou abaixo dos demais sítios de plantio, fato que não ocorreu nos quatro primeiros meses (Tabela 1). Aparentemente, a alta temperatura e a baixa umidade apresentada em Carlos Chagas foram responsáveis por proporcionar ambiente com DPV superior ao dos outros sítios de plantio (Figura 1). De acordo com White et al. (2009), a realização de desbastes em plantios de eucalipto pode diminuir os danos causados pela falta de água no solo. 
Tabela 6 - Massa seca de raízes finas (PSRF), médias (PSRM) e grossas (PSRG) de plantas clonais de Eucalyptus grandis $\mathrm{x}$ E. urophylla, em diferentes sítios, seis meses após o plantio.

Table 6 - Fine roots dry mass (PSFR), average size (PSRM) and thick roots (PSRG) of clonal Eucalyptus gtrandis x $\boldsymbol{E}$. urophylla plants in deifferent sites six months after the planting.

\begin{tabular}{|c|c|c|c|c|}
\hline \multicolumn{5}{|c|}{ PSRF (g) } \\
\hline Tratamento & Aracruz & Mucuri & Montanha & Carlos Chagas \\
\hline AG & 44.62 а B & $17.32 \mathrm{~b} \mathrm{~A}$ & $13.30 \mathrm{~b} \mathrm{~A}$ & $9.44 \mathrm{~b} \mathrm{~A}$ \\
\hline $\mathrm{AM}$ & 43.18 a $\mathrm{B}$ & $24.24 \mathrm{ab} \mathrm{A}$ & 9.57 b A & $8.03 \mathrm{~b} \mathrm{~A}$ \\
\hline MG & 66.82 a A & 30.33 b A & $11.28 \mathrm{~b} \mathrm{~A}$ & $11.02 \mathrm{~b} \mathrm{~A}$ \\
\hline MM & 55.29 а $\mathrm{AB}$ & $18.71 \mathrm{~b} \mathrm{~A}$ & $11.51 \mathrm{~b} \mathrm{~A}$ & 9.59 b A \\
\hline $\mathrm{PM}$ & 47.08 a $\mathrm{AB}$ & 35.66 а A & $11.11 \mathrm{~b} \mathrm{~A}$ & $9.47 \mathrm{~b} \mathrm{~A}$ \\
\hline $\mathrm{PF}$ & 37.31 a B & 30.65 а A & 7.97 b A & $8.64 \mathrm{~b} \mathrm{~A}$ \\
\hline \multicolumn{5}{|c|}{$\mathrm{CV}(\%)=65,65$} \\
\hline \multicolumn{5}{|c|}{ PSRM (g) } \\
\hline Tratamento & Aracruz & Mucuri & Montanha & Carlos Chagas \\
\hline AG & 142.33 a $\mathrm{A}$ & $47.90 \mathrm{~b} \mathrm{~A}$ & $21.91 \mathrm{~b} \mathrm{~A}$ & $25.88 \mathrm{~b} \mathrm{~A}$ \\
\hline $\mathrm{AM}$ & 50.49 а B & 53.37 a $\mathrm{A}$ & 31.27 a $\mathrm{A}$ & 25.41 a $\mathrm{A}$ \\
\hline MG & 65.15 а $\mathrm{AB}$ & 37.35 а $\mathrm{A}$ & 24.33 a $\mathrm{A}$ & 32.63 a $\mathrm{A}$ \\
\hline MM & 52.37 a $\mathrm{B}$ & 44.06 a $\mathrm{A}$ & 42.03 a $\mathrm{A}$ & 18.57 a $\mathrm{A}$ \\
\hline $\mathrm{PM}$ & 49.99 а $\mathrm{B}$ & 35.54 a A & 31.62 а A & 11.22 a $\mathrm{A}$ \\
\hline $\mathrm{PF}$ & 40.00 a B & 46.68 a $\mathrm{A}$ & 37.96 a A & 16.57 a A \\
\hline \multicolumn{5}{|c|}{ CV $(\%)=178,04$} \\
\hline \multicolumn{5}{|c|}{ PSRG (g) } \\
\hline Tratamento & Aracruz & Mucuri & Montanha & Carlos Chagas \\
\hline AG & 1172.96 а $\mathrm{AB}$ & 955.89 а $\mathrm{A}$ & 806.23 a $\mathrm{A}$ & 630.29 a A \\
\hline $\mathrm{AM}$ & 1140.50 а $\mathrm{AB}$ & 779.12 а $A$ & 937.76 а $\mathrm{A}$ & $227.02 \mathrm{~b} \mathrm{~A}$ \\
\hline MG & 1365.49 а A & 841.08 ab A & 683.45 b A & 452.16 b A \\
\hline MM & 915.26 ab АB & $629.66 \mathrm{ab} \mathrm{A}$ & 1172.33 a A & 503.18 b A \\
\hline $\mathrm{PM}$ & 1290.55 а АВ & 783.09 ab A & 797.23 ab A & 340.37 b A \\
\hline $\mathrm{PF}$ & 695.02 а B & 682.43 а A & 865.50 a $\mathrm{A}$ & 415.03 a $\mathrm{A}$ \\
\hline
\end{tabular}

Médias seguidas pelas mesmas letras minúsculas na linha e maiúscula na coluna não diferem entre si, pelo teste de Duncan a $5 \%$ de probabilidade.

Mudas altas (A) 31 a 40 cm; médias (M) 21 a 30 cm; pequenas (P) 10 a 20 cm; mudas grossas (G) 3,1 a 4,0 mm; médias (M) 2,1 a 3 mm; e finas (F) 1,5 a 2,0 mm. Raízes grossas: diâmetro acima de $5 \mathrm{~mm}$; raízes médias: diâmetro entre 2,5 e 4,99 mm e raízes finas com diâmetro abaixo de 2,49 mm.

O DPV é variável que leva em consideração os valores de temperatura e umidade relativa do ar, apresentando relação direta com o funcionamento de abertura e fechamento estomático que, por sua vez, está relacionado ao processo fotossintético da planta. Dessa forma, o fechamento estomático atua reduzindo a perda de água pela planta (CHAVES, 1991; LAWLOR, 1995). Consequentemente, a difusão do $\mathrm{CO}_{2}$ no interior da folha é diminuída (FARQUHAR; SHARKEY, 1982). A diminuição da concentração interna do $\mathrm{CO}_{2}$ resulta na limitação da fotossíntese (CORNIC et al., 2000) e, consequentemente, a diminuição do crescimento.

\section{CONCLUSÕES}

- Mudas com maiores dimensões de altura e diâmetro apresentaram maior crescimento inicial, mas as diferenças tenderam a desaparecer ao longo do tempo.

- Em Carlos Chagas houve menor produção de raízes pelas plantas que nos demais sítios.

- Mudas altas e grossas tiveram maior produção inicial de biomassa de folhas, lenho, casca e galhos, em todos os sítios.

- O sítio de Carlos Chagas apresentou as condições mais adversas para o crescimento após o plantio.

Revista Árvore, Viçosa-MG, v.35, n.1, p.01-11, 2011 


\section{AGRADECIMENTOS}

- À Universidade Estadual do Norte Fluminense Darcy Ribeiro.

À empresa Fibria Celulose.

\section{REFERÊNCIAS}

ALFENAS, A. C. et al. Clonagem e doenças do eucalipto. Viçosa, MG: Universidade Federal de Viçosa, 2004. 442p.

BARNETT, J. P. Relating seedling morphology of container grown southern pines to field sucess. In: CONVENTION OF THE SOCIETY OF AMERICAN FORESTERS (1983: Portland) Proceedings... New Orleans: USDA Forrest Service, Southern Forest Experiments Station, 1983. p.405-407.

BARROSO, D. G. et al. Regeneração de raízes de mudas de eucalipto produzidas em diferentes recipientes e substratos. Scientia Agrícola, v.57, n.2, p.229-237, 2000a.

BARROSO, D. G. et al. Efeitos do recipiente sobre o desenvolvimento pós-plantio de Eucalyptus camaldulensis e E. urophylla. Revista Árvore, v.24, n.3, p.291-296, 2000 b.

BARROSO, D. G. et al. Efeitos do recipiente sobre o desempenho pós-plantio de Eucalyptus camaldulensis e E. urophylla. Revista Árvore, v.21, n.3, p.291-296, 2000c.

BERGER, R. et al. Efeito do espaçamento e da adubação no crescimento de um clone de Eucalyptus saligna Smith. Ciência Florestal, v.12, n.2, p.75-87, 2002.

BÖHM, W. Methods of studying root systems. Berlin: Springer-Verlag, 1978.188p.

BORGES, R. C. G. et al. Correlações entre caracteres de crescimento em Eucaliyptus grandis S. Hill ex Maiden. Revista Árvore, v.4, n.2, p.146-156, 1980.

CARneiro, J. G. A. Produção e controle de qualidade de mudas florestais. Curitiba: UFPR/FUPEF; Campos: UENF, 1995. 451p.

Revista Árvore, Viçosa-MG, v.35, n.1, p.01-11, 2011
CARNEIRO, J. G. A.; RAMOS, A. Influência da altura aérea, diâmetro de colo e idade de mudas de Pinus taeda sobre a sobrevivência e desenvolvimento após 15 meses e aos seis anos após o plantio. In: SEMINÁRIO DE SEMENTES E VIVEIROS FLORESTAIS, 1., 1981, Curitiba. Anais... Curitiba: FUPEF, 1981. p.91-110.

CHAVES, M. M. Effects of water deficits on carbon assimilation. Journal of Experimental Botany, v.42, n.234, p.1-16, 1991.

CORNIC, G. Drought stress inhibits photosynthesis by decreasing stomatal aperture not by affecting ATP synthesis. Trends in Plant Science, v.5, n.5, p.187-188, 2000.

FARQUHAR, G. D.; SHARKEY, T.D. Stomatal condutance and photosynthesis. Annual Review of Plant Physiology, v.33, p.317-45, 1982.

FREITAS, T. A. Sistema de blocos prensados para produção de mudas de eucalipto. 2003. 94f. Dissertação (Mestrado em Produção Vegetal) - Universidade Estadual do Norte Fluminense Darcy Ribeiro, Campos dos Goytacazes, 2003.

JOSÉ, A. C.; DAVIDE, A. C.; OLIVEIRA, S. L. Produção de mudas de aroeira (Schinus terebinthifolius Raddi) para recuperação de áreas degradadas pela mineração de bauxita. Cerne, v.11, n.2, p.187-196, 2005.

LARCHER, W. Ecofisiologia vegetal. São Paulo: Rima Artes e Textos, 2000. 531p.

LAWLOR, D.W. Photosynthesis, productivity and environment. Journal of Experimental Botany, v.46, p.1449-1461, 1995.

LELES, P. S. S. et al. Qualidade de mudas de Eucalyptus spp. produzidas em blocos prensados e em tubetes. Revista Árvore, v.24, n.1, p.13-20, 2000.

MORGADO, I. F. et al. Nova metodologia de produção de mudas de Eucalyptus grandis W. Hill ex Maiden utilizando resíduos prensados como substratos. Revista Árvore, v.24, n.1, p.27-33, 2000.

NAVES-BARBIERO, C. C. et al. Fluxo de seiva e condutância estomática de duas espécies lenhosas sempre-verde no campo sujo e serrado. Revista Brasileira de Fisiologia Vegetal, v.12, n.2, p.119-134, 2000.

Revista Árvore, Viçosa-MG, v.35, n.1, p.01-11, 2011 
PARVIAINEN, J. V. Qualidade e avaliação da qualidade de mudas florestais. In: SEMINÁRIO DE SEMENTES E VIVEIROS FLORESTAIS, 1., 1981, Curitiba. Anais... Curitiba: FUPEF, 1981. p.59-90.

RESENDE, J. L. P.; LIMA Jr, V. B.; SILVA, M. L. O setor florestal brasileiro. Informe

Agropecuário, v.18, n.185, p.7-14, 1996.

SANTOS, C. B. et al. A Efeito do volume de tubetes e tipos de substratos na qualidade de mudas de Cryptomeria japonica (L. F.) D.

Ciência Florestal, v.10, n.2, p.1-15, 2000.

SOCIEDADE BRASILEIRADE SILVICULTURASBS. Dados estatísticos sobre a produção nacional de eucalipto. Sociedade Brasileira de Silvicultura. Disponível em: http://www.sbs.org.br/secure/ estatisticas.htm. Acesso em 29 de jan. 2006.
SOUZA, M. J. H. et al.Disponibilidade hídrica do solo e produtividade do eucalipto em três regiões da bacia do Rio Doce. Revista Árvore, v.30, n.3, p.399-410, 2006.

TAIZ, L.; ZEIGER, E. Fisiologia vegetal. 3.ed. Porto Alegre: Artmed, 2004. 719p.

VOGT, K. A.; PERSSON, H. Measuring growth and development of roots. In: LASSOIE, J. P.; HiNCKLEY, T. M. (Eds.). Techniques and approaches in forest tree ecophysiology. Boca Raton: CRC Press, 1991. p.447-501.

WHITE, D. A. et al. Managing productivity and drought risk in Eucalyptus globulus plantations in south-western Austrália. Forest Ecology and Management, v.259, p.33-44, 2009. 
